\title{
Prophylactic human papillomavirus vaccines
}

\author{
Douglas R. Lowy and John T. Schiller
}

\author{
Laboratory of Cellular Oncology, Center for Cancer Research, National Cancer Institute, NIH, Bethesda, Maryland, USA.
}

\begin{abstract}
Human papillomavirus (HPV) infection causes virtually all cases of cervical cancer, the second most common cause of death from cancer among women worldwide. This Review examines prophylactic HPV subunit vaccines based on the ability of the viral L1 capsid protein to form virus-like particles (VLPs) that induce high levels of neutralizing antibodies. Following preclinical research by laboratories in the nonprofit sector, Merck and GlaxoSmithKline are developing commercial versions of the vaccine. Both vaccines target HPV16 and HPV18, which account for approximately $70 \%$ of cervical cancer. The Merck vaccine also targets HPV6 and HPV11, which account for approximately $90 \%$ of external genital warts. The vaccines have an excellent safety profile, are highly immunogenic, and have conferred complete type-specific protection against persistent infection and associated lesions in fully vaccinated women. Unresolved issues include the most critical groups to vaccinate and when the vaccine's cost may be low enough for widespread implementation in the developing world, where $80 \%$ of cervical cancer occurs.
\end{abstract}

\section{Human papillomavirus infection and disease}

Of the 10 million cases of cancer that develop annually throughout the world, more than $15 \%$ are estimated to be attributable to infectious agents (1). Infection by human papillomaviruses (HPVs) accounts for approximately $30 \%$ of these cancers $(-5 \%$ of all cancers), with hepatitis B and C viruses and Helicobacter pylori together accounting for another $60 \%$ of cancers with an infectious etiology.

HPVs infect the stratified squamous epithelia of skin and mucous membranes, where they cause benign lesions, some of which have the potential to progress to invasive cancer. (2-4). HPVs are small, nonenveloped viruses whose approximately $8-\mathrm{kb}$ circular genome encodes 2 structural proteins, L1 and L2, that form the viral capsid, plus several nonstructural proteins that are important for the virus life cycle but are not incorporated into virions. To establish infection, microtrauma or erosion of the overlying epithelial layers is thought to enable HPVs to infect cells of the basal epithelial layer, where the stem cells and other long-lived cells are found (Figure 1). HPV infections tend to last months or years because the viral genome successfully parasitizes these cells and because the virus evades the immune system by limiting most viral gene expression and viral replication to suprabasal cell layers. Most infections are self-limited, presumably because the host eventually mounts a successful immune response.

The benign lesions induced by HPVs include nongenital and anogenital skin warts, oral and laryngeal papillomas, and anogenital mucosal condylomata (Figure 2). Anogenital infections are almost always transmitted sexually. Long-term infection by a subset of HPVs can lead to malignant anogenital tumors, including cancers of the anus, penis, vulva, vagina, and cervix (5-7). A proportion of oral cancer is also attributable to $\operatorname{HPV}(8,9)$. While HPV infection has been associated on limited occasions with esophageal cancer and skin cancer, a frequent causal link, although plausible, remains more tenuous $(6,10)$.

Among the cancers attributable to HPV infection, cervical cancer has received the most attention (11), as it accounts for about $10 \%$ of all cancers in women worldwide. Cervical cancer is the sec-

Nonstandard abbreviations used: CIN, cervical intraepithelial neoplasia; HPV, human papillomavirus; HSV, herpes simplex virus; VLP, virus-like particle.

Conflict of interest: The authors, as employees of the National Cancer Institute, $\mathrm{NIH}$, are inventors of the HPV VLP vaccine technology described in this Review. The technology has been licensed by the NIH to the 2 companies, Merck and GlaxoSmithKline, that are developing the commercial HPV vaccines described herein.

Citation for this article: J. Clin. Invest. 116:1167-1173 (2006). doi:10.1172/JCI28607. ond most common cause of death from cancer, after breast cancer, among women worldwide. The interval between the acquisition of HPV infection and malignant progression usually takes at least 10 years (Figure 3 ) and is frequently longer $(7,12)$. Cervical cancer is therefore very uncommon in women under 25 ; the incidence rises progressively for women over 25 and is highest for women over 40. About $80 \%$ of cervical cancers occur in less-developed countries, primarily because they lack sufficient resources for high-quality cervical cancer screening programs that detect cervical abnormalities via Pap smear testing or testing for the presence of cervical HPV DNA (13). Virtually all cases of cervical cancer are attributable to sexually acquired HPV infection (14), while infection by HPV accounts for a variable proportion of the other tumors in which the virus has been implicated etiologically. Of all cancer cases linked etiologically to HPV, cervical cancer accounts for about two-thirds of them. This cancer can result from infection by any 1 of about 15 oncogenic HPV types, but HPV16 and HPV18 predominate, accounting for about $50 \%$ and $20 \%$ of cervical cancer, respectively (15). These 2 types account for an even higher proportion of the other genital and mucosal cancers attributable to HPV infection (6).

Genital HPV infection is believed to be the most common sexually transmitted viral infection, with an estimated prevalence of about $20-40 \%$ among sexually active 20 -year-old women, an estimated 3-year cumulative incidence of more than $40 \%$ in studies of college women in the United States, and an estimated lifetime risk for women of at least $75 \%$ for one or more genital HPV infections $(16,17)$. Most genital HPV infections are benign, subclinical, and self-limited, and a high proportion of infections associated with low-grade cervical dysplasias (Figure 2) also regress spontaneously $(7,17)$. By contrast, persistent cervical infection (often defined as an infection that is detected more than once in an interval of 6 months or longer) with an oncogenic HPV type, especially HPV16 and HPV18, is the most important risk factor for progression to high-grade dysplasia (Figure 2) (18), which is recognized as a precancerous lesion that should be treated to prevent the development of invasive cancer. Locally, ablative therapy is used successfully to treat high-grade dysplasia (13).

\section{Prophylactic HPV vaccine: development and efficacy studies}

Identification of a viral agent such as HPV as a cause of disease(s) implies that successful prophylactic or therapeutic intervention 


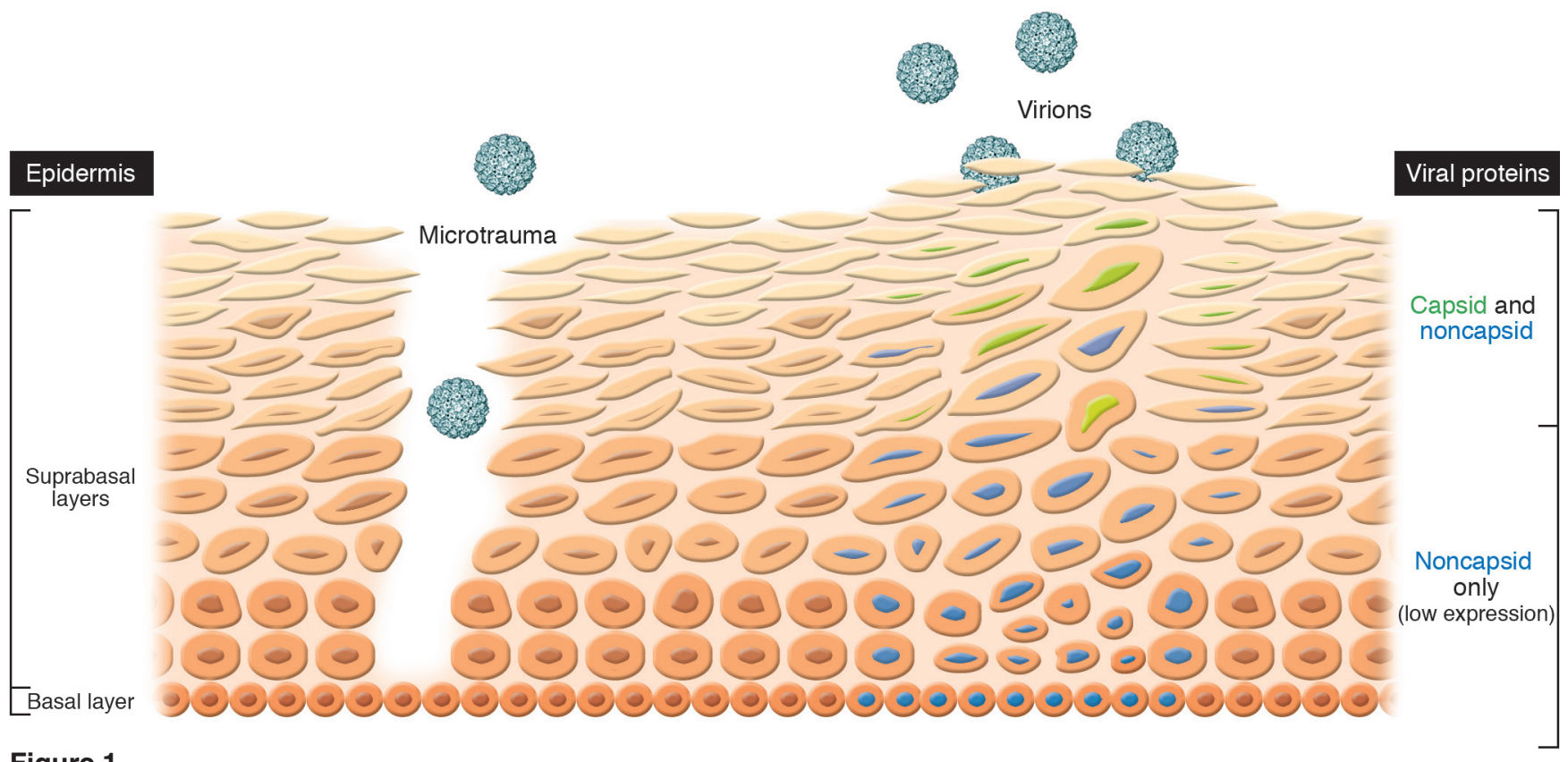

Figure 1

Papillomavirus life cycle. To establish infection, the virus must infect basal epithelial cells that are long lived or have stem cell-like properties. Microtrauma to the suprabasal epidermal cells probably enables the virus to infect the cell within the basal layer. The viral genome maintains itself as an episome in basal cells, where the viral genes are poorly expressed. Viral replication takes place in suprabasal layers and is tied to the epidermal differentiation process. The presence of the virus causes morphological abnormalities in the epithelium, including papillomatosis, parakeratosis, and koilocytosis. Progeny virus is released in desquamated cells.

against the viral agent should prevent the disease(s) it causes. A preexisting viral infection can theoretically be targeted by an antiviral or a therapeutic vaccine. Successful antivirals have been developed for the treatment of some viral infections, including diseases such as HIV and influenza (19), but not against viruses such as HPV. A therapeutic vaccine against HPV infection would be highly desirable to prevent the cancer-associated complications of HPV infection, which only develop after many years of infection. However, despite ongoing efforts to develop effective therapeutic vaccines against HPV and other viral infections, none has been shown to be highly effective clinically (20), probably because the vaccines have not yet adequately mimicked critical aspects of a curative immune response. On the other hand, prophylactic vaccines have been developed against a variety of human viral pathogens and are often a cost-effective approach to interfere with the diseases caused by these pathogens (21). To be widely implemented, a prophylactic vaccine generally needs to confer high-level protection for at least several years without boosting and to be particularly safe, as it is given to healthy individuals.

The recognition of HPV as the cause of cervical cancer and other diseases therefore implied that an effective HPV vaccine should be able to interfere with the benign and malignant conditions attributable to HPV infection. However, approved prophylactic vaccines have been directed against infectious agents that cause systemic disease, and efforts to develop vaccines against sexually transmitted agents such as HPV whose disease results from local infection had not proven successful. It is believed that neutralizing antibodies form the cornerstone of most prophylactic vaccines, and viruses that cause disease only after passing through the circulation are accessible to the neutralizing antibodies pres- ent in the blood (22). Another limitation was that the presence of oncogenes in HPVs suggested that a subunit vaccine approach would theoretically be preferable to an inactivated vaccine or an attenuated live-virus vaccine, and it was unclear whether a subunit $\mathrm{HPV}$ vaccine would have the potential to be effective against the local infections caused by genital HPVs.

Despite these uncertainties, 2 pharmaceutical companies, Merck and GlaxoSmithKline, have recently reported a remarkable degree of protection by candidate prophylactic HPV vaccines (see below). The vaccines that both companies are developing are subunit virus-like particle (VLP) vaccines composed of a single viral protein, L1, which is the major structural (capsid) protein of the virus and contains the immunodominant neutralization epitopes of the virus. The vaccines are based primarily on preclinical research showing that (a) when expressed in cells, L1 has the intrinsic ability to self-assemble into VLPs (Figure 4) (23-26) that can induce high levels of neutralizing antibodies $(23,27,28)$; (b) in animal models of animal papillomavirus infection, parenteral vaccination with L1 VLPs protects from high-dose challenge with homologous virus (29-31), while animals are not protected by systemic immunization with denatured L1 or L1 VLPs from a heterologous papillomavirus because L1 neutralization epitopes are conformationally dependent and predominantly type specific (29); and (c) protection can be passively transferred by immune $\operatorname{IgG}(29,30)$. The VLPs from the Merck vaccine are produced in yeast (32-34), while the VLPs from the GlaxoSmithKline vaccine are produced in insect cells via recombinant baculovirus (35). Merck uses alum as an adjuvant in its vaccine, while GlaxoSmithKline uses AS04, a proprietary adjuvant composed of alum plus monophosphoryl lipid A (a detoxified form of lipopolysaccharide). Both vaccines use 


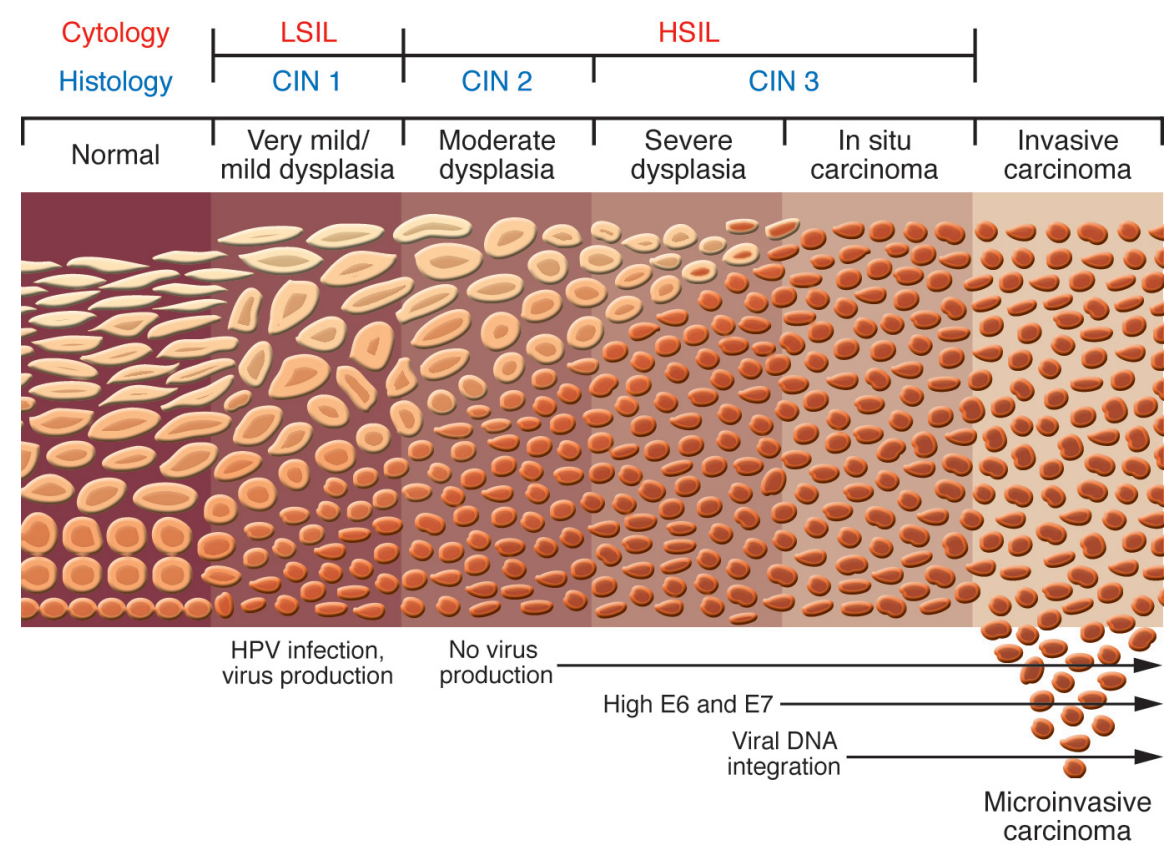

\section{Figure 2}

Progression from a benign cervical lesion to invasive cervical cancer. Infection by oncogenic HPV types, especially HPV16, may directly cause a benign condylomatous lesion, lowgrade dysplasia, or sometimes even an early high-grade lesion. Carcinoma in situ rarely occurs until several years after infection. It results from the combined effects of HPV genes, particularly those encoding $\mathrm{E} 6$ and $\mathrm{E} 7$, which are the 2 viral oncoproteins that are preferentially retained and expressed in cervical cancers; integration of the viral DNA into the host DNA; and a series of genetic and epigenetic changes in cellular genes. HSIL, highgrade squamous intraepithelial lesion; LSIL, low-grade squamous intraepithelial lesion. purified particles, which are given as 3 intramuscular injections over a 6-month period.

Of course, it would be desirable for an HPV vaccine to have the ability to prevent all cases of cervical cancer. However, although the 15 oncogenic types implicated in cervical cancer are more closely related to each other phylogenetically than they are to the HPVs that cause nongenital skin lesions (warts) (36), the immunodominant epitopes in L1 VLPs induce neutralizing antibodies that are predominantly type specific (37). It has therefore been necessary, at least for the first generation HPV vaccines, to focus on the HPV types found most frequently in cervical cancer. This consideration has led both companies to focus on HPV16 and HPV18, which, as noted above, account for about $70 \%$ of cases of cervical cancer. The Merck vaccine also targets HPV6 and HPV11, which together account for about $90 \%$ of external genital warts (38); the latter 2 types also infect the cervix, but are not implicated in cervical cancer. Thus, the GlaxoSmithKline vaccine that is currently in phase III trials is a bivalent vaccine composed of VLPs from HPV16 and HPV18, while the vaccine that Merck has used for its phase III trials is a quadrivalent vaccine that contains VLPs from HPV6, HPV11, HPV16, and HPV18.

When considering appropriate end points for determining vaccine efficacy, the most relevant end points recommended by an FDA vaccine advisory panel (39) were a reduction in the incidence of vaccine type-specific persistent infections and of associated moderate- and high-grade cervical dysplasias and carcinomas in situ, which together are referred to as cervical intraepithelial neoplasia 2+ (CIN2+; CIN is graded as CIN1, CIN2, and CIN3 for low-, moderate-, and high-grade dysplasia, respectively). HPV DNA testing results have been shown to be substantially more reproducible than the pathological diagnosis of dysplasia (40), but moderateand high-grade dysplasias represent clinical end points that trigger therapeutic intervention. It is important to note that it would be unethical to use cervical cancer as a primary end point for vaccine efficacy trials, as cervical cancer screening can prevent the vast majority of cancer through the identification of precancers, which are then treated. Also, the interval between infection and the development of invasive cancer usually takes more than 10 years $(7,12)$.

Following the observations that systemic vaccination with a monovalent HPV16 L1 VLP vaccine was safe and highly immunogenic (41), even without adjuvant, a Merck-sponsored proofof-principle efficacy trial of an HPV16 L1 VLP vaccine reported that fully vaccinated women who were HPV negative throughout the vaccination period were completely protected against the development of persistent incident infection with HPV16 when followed for an average of 17 months (Table 1 and ref. 32). This vaccine cohort has now been followed for an average of 3.5 years, and the high level of protection was maintained throughout this period (Table 1 and ref. 34). After the initial peak, the levels of serum antibodies appeared to decline approximately 10 -fold over the first 2 years following vaccination but then remained stable for the remainder of the follow-up period. If the level of serum antibodies represents a surrogate for protection against infection, the stability of the antibody titers would suggest that high-level protection may continue substantially beyond 3.5 years. Preliminary efficacy results from Merck's quadrivalent vaccine were also published and showed excellent protection against the viruses targeted by the vaccine (Table 1 and ref. 33). In unpublished studies, Merck has reported at scientific meetings on the clinical efficacy of the multinational phase III trial of their quadrivalent vaccine, with an average follow-up of 1.5 years $(42,43)$. When fully vaccinated women who remained negative for infection throughout the vaccination period were analyzed against the comparable placebo cohort, the vaccine was $100 \%$ effective in preventing CIN2+ associated with HPV16 or HPV18 and also in preventing external genital warts associated with HPV6 or HPV11. Even when the efficacy was compared starting 1 month after the first immunization, protection for these end points was greater than $90 \%$. Thus systemic immunization with a subunit HPV vaccine can achieve a high degree of protection in women against benign and premalignant diseases induced by this sexually transmitted local infection of the genital mucosa or skin. 


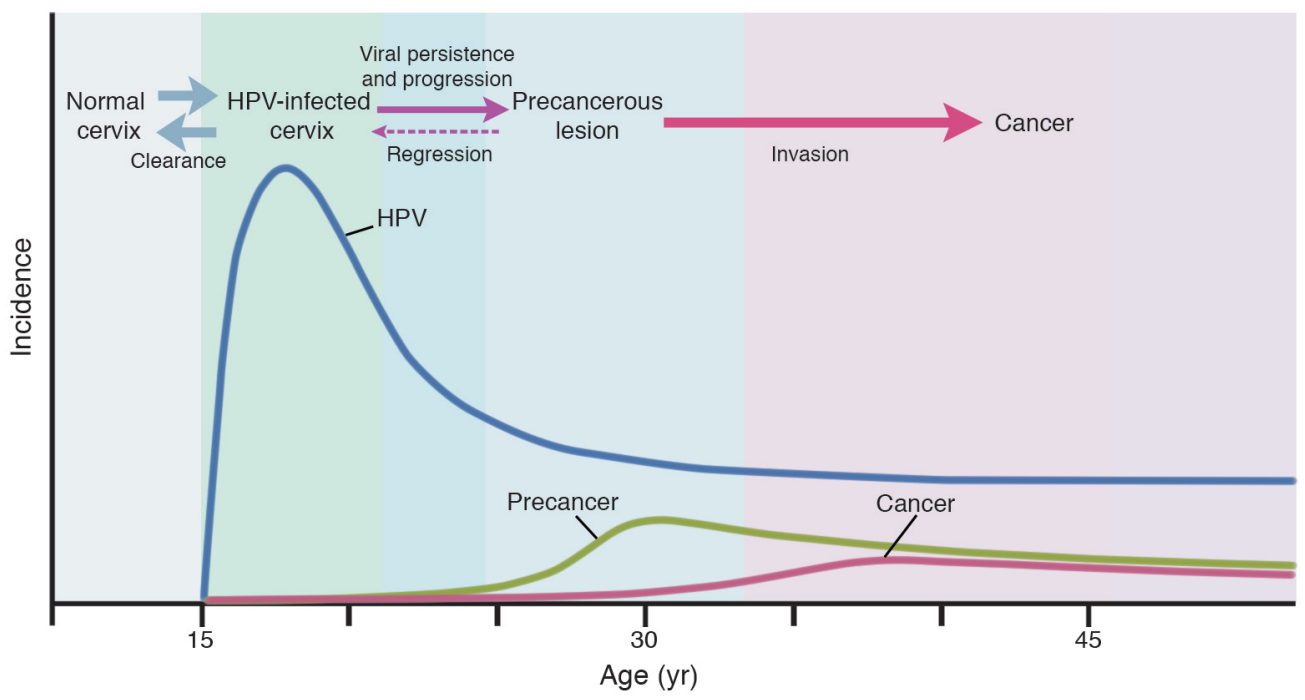

Figure 3

Relationship among incidences of cervical HPV infection, precancer, and cancer. The HPV curve emphasizes the high incidence of infection that develops soon after women initiate sexual activity and subsequent lower incidence because a high proportion of infections are self-limited. The precancer incidence curve follows several years behind the HPV incidence curve and is substantially lower than that of HPV incidence, as there is generally a delay between the acquisition of HPV infection and precancer development, and only a subset of infected women develop precancers. The cancer incidence curve follows several years behind the precancer curve, reflecting the relatively long interval between precancer and progression to invasive cancer. As women approach 40 years of age, the incidence of cancer begins to approach the incidence of precancer. Figure modified with permission from the New England Journal of Medicine (53).

The Merck-sponsored trials have not reported on the comparative incidence of infection by other HPV types not included in the vaccines. However, the proof-of-principle monovalent HPV16 trial has reported on the total number of patients with various grades of cervical dysplasia, and whether the dysplasias were associated with HPV16, for the fully vaccinated women who were HPV negative throughout the vaccination period (32). In contrast to the complete protection seen against HPV16-associated dysplasias, there was no difference between the placebo and vaccinated groups in the number of non-HPV16-associated dysplasias in the initial report on this cohort. In the 3.5-year follow-up report (34), there was also no evidence that non-HPV16-associated dysplasias in the vaccinated group were less frequent in number than those in the nonvaccinated group. These results strongly suggest that the protection conferred by Merck HPV16 monovalent vaccine was predominantly HPV type specific.

GlaxoSmithKline has also sponsored a proof-of-principle efficacy trial of an HPV16 and HPV18 bivalent vaccine similar to the one that is currently in phase III trials (Table 1 and ref. 35). Their placebo-controlled proof-of-principle trial had a smaller number of participants who were followed for an average of 18 months. In the group of women who were fully vaccinated and remained uninfected throughout the vaccination period, all subsequent persistent infections associated with HPV16 and HPV18 occurred in the placebo group, although there were only a total of 7 cases (5 with HPV16 and 2 with HPV18). When incident persistent infection was monitored starting 1 month after the first dose of vaccine, combined protection against HPV16 and HPV18 was still about $90 \%$. Another potentially important result, which has been presented at meetings but not yet published, is that the vaccine has been associated with some cross-protection against HPV types closely related to HPV16 and HPV18, although this protection was less complete than that offered against HPV16 and HPV18 (44, 45). As Merck has not reported results analyzed in this manner, it is difficult to know whether the cross-protection represents an activity that may be greater in the GlaxoSmithKline vaccine. It will be important for the ongoing large-scale efficacy trials to analyze this parameter, as cross-protection against HPV types not in the vaccine could enhance its overall utility.

\section{Vaccine implementation issues}

The notable efficacy results (Table 1) have thus far been correlated with an excellent safety profile, which make it likely that one or both vaccines will be licensed in the near future. In fact, Merck applied to the FDA in December 2005 for a license to sell their quadrivalent vaccine. It is anticipated that application for licensure will also come from GlaxoSmithKline if their phase III trial results are similarly positive.

Several issues about the vaccine remain to be addressed. It will be important to confirm that the strong safety profile remains intact as more individuals receive the vaccine. As noted above, it remains to be determined how long the high level of type-specific protection is maintained, as this issue will have implications for whether and when booster injections might be advisable and will also contribute to the cost-effectiveness of the vaccine. It remains to be seen whether the cross-protection against HPV types not in the vaccine, as noted in the GlaxoSmithKline proof-of-principle trial, will be confirmed in the large-scale trials of this vaccine and whether the Merck vaccine possesses similar properties. A substantial degree of cross-protection could increase the potential impact of the vaccine by further reducing the incidence of serious genital HPV infections and by reducing the number of abnormal Pap tests and the cost of their follow-up. On the other hand, protection against heterologous HPV types is likely to wane more quickly than that 


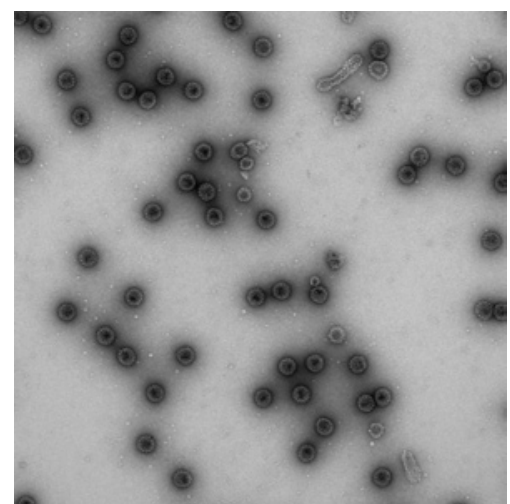

\section{Figure 4}

Electron micrograph of HPV16 L1 VLPs. Original magnification, $\times 14,500$. Image courtesy of Yuk-Ying Susana Pang (Laboratory of Cellular Oncology, National Cancer Institute).

against the HPV types specifically targeted by the vaccine, which could also have implications for boosting.

Another unanswered question is whether vaccination might alter the natural history of prevalent HPV infection by reducing the incidence of persistent infection or cytological abnormalities. The large vaccine trials may have a sufficient number of prevalent infections attributable to the HPV types in the vaccine to address this question. If such a "therapeutic effect" were seen, it could provide an added rationale to vaccinate sexually active women who might have prevalent infection with one of the types in the vaccine. The most likely explanation for such an effect would be that the vaccine had reduced the efficiency of transmission of an early infection from one genital site to other genital sites, presumably via specific antibodies in the genital tract (46). It is also possible that the vaccine could have direct therapeutic effects against established lesions. However, this possibility seems less likely, as persistent infection is usually attributed to the presence of the viral genome in long-lived basal epithelial cells, which do not express L1. The experimental evidence also does not support this possibility: when the effect of the VLP vaccine was tested on established lesions in an animal model (bovine papillomavirus type 4 [BPV-4], which induced benign oral mucosal lesions), it did not induce their regression, although the vaccine was very effective when given prophylactically (31).

Although the immune response of men to the vaccine is similar to that of women (41), it is not yet known whether the vaccine will be protective in men. Many vaccines have comparable efficacy in males and females. However, a subunit vaccine for type 2 herpes simplex virus (HSV gD vaccine), another sexually transmitted viral infection, was found to be effec-

\section{Table 1}

tive in women but not in men, which raises the possibility that an analogous difference might be seen with the HPV vaccine (47). As HSV infection is more likely to be mucosal in women and cutaneous in men, it was speculated that the difference in protection from the HSV vaccine might be attributable to higher antibody titers in mucosa than in skin. Even if this explanation is relevant to HSV, the high level of protection of the Merck vaccine against cutaneous genital warts in women would be expected to apply to men as well, since these warts appear on cornified skin in both sexes. It may also be relevant that the protection induced in women by the HSV vaccine was less robust than that induced by the HPV vaccine (47). Efficacy trials of the HPV vaccine in men will directly address this issue.

A key question will be whom to vaccinate. In the United States, the main national advisory committee will be the Advisory Committee on Immunization Practices at the Centers for Disease Control and Prevention. Until there are data that show the vaccine is protective in men, it would seem most logical to focus public health efforts primarily on vaccinating women. If the principal activity of the vaccine is the prevention of incident HPV infection, the greatest reduction in the number of infections would likely result from immunizing girls or women before they become sexually active. In the United States, this consideration would imply that pre- or young adolescent girls would be prime candidates for the vaccine. Of course, older girls and women with no prior sexual exposure should also achieve maximum benefit from the vaccine, which implies that "catch-up" vaccination for these groups should be seriously considered. Giving the vaccine to women who have had some prior sexual activity could also reduce their number of infections, although their degree of benefit from the vaccine would probably be inversely related to their degree of prior sexual activity. If the vaccines are shown to be highly effective in men, it will be logical to include them in vaccination programs in highresource settings such as the United States, especially if the Merck vaccine is able to protect men against genital warts. From a public health perspective, however, the degree to which vaccination of males would contribute to herd immunity is unclear. In some models of sexually transmitted infections, if a high proportion of one gender is vaccinated and the vaccine is effective in preventing transmission, vaccinating the other gender achieves a relatively

Proof-of-principle HPV VLP prophylactic efficacy trials

$\begin{array}{lcc}\text { Study } & \text { Koutsky et al. (32) } & \text { Harper et al. (35) } \\ \text { HPV VLP type } & 16 & 16,18 \\ \text { Adjuvant } & \text { Alum } & \text { AS04 } \\ \text { Sponsor } & \text { Merck } & \text { GSK } \\ \text { Trial site } & \text { United States } & \text { United States, } \\ & & \text { Canada, Brazil } \\ \text { Subject age } & 16-23 & 15-25 \\ \text { No. subjects (ATP) } & 1,533 & 721 \\ \text { Vaccination schedule (mo) } & 0,2,6 & 0,1,6 \\ \text { Follow-up (yr) } & 1.5 & 1.5 \\ \text { Persistent infections }{ }^{A} & 42 / 0(100) & 7 / 0(100) \\ \text { CIN1+D } & 9 / 0(100) & 6 / 0(100)\end{array}$

Villa et al. (33)

$6,11,16,18$

Alum

Merck

United States,

European Union, Brazil

$16-23$

468

$0,2,6$

2.5

$36 / 4^{B}(90)$

$3 / 0(100)$
Mao et al. (34)

16

Alum

Merck

United States

$16-23$

1,505

$0,2,6$

3.5

$111 / 7^{\mathrm{C}}(94)$

$24 / 0(100)$

Shown are according-to-protocol (ATP) analyses for the HPV types included in the vaccines. ${ }^{A}$ Values are shown as number of controls versus number of vaccinees with persistent infections; values in parentheses indicate percent efficacy. ${ }^{B}$ Ten of 36 controls and 3 of 4 vaccinees were HPV DNA positive only at the last visit. CNineteen of 111 controls and 7 of 7 vaccinees were HPV DNA positive only at the last visit. DValues are shown as number of controls versus number of vaccinees that were CIN1+; values in parentheses indicate percent efficacy. GSK, GlaxoSmithKline. 
small increase in herd immunity (48). In addition, more than $80 \%$ of cancers attributable to HPV infection occur in women (1). These considerations suggest that vaccination of women should probably have the highest priority in settings with limited resources.

\section{Anticipated impact of vaccination}

The previous section has noted several important unanswered questions that make it difficult to predict the actual impact of a vaccine on HPV infection and disease. However, it is possible to give some estimates if it is assumed that the vaccine will provide at least $90 \%$ type-specific efficacy, with boosters as necessary to ensure that this protection is of long duration. Under those circumstances, the greatest short-term impact in industrialized nations such as the United States would be a reduction in the overall number of CIN2+ cases to about one-third to one-half as many such lesions in vaccinated women compared with nonvaccinated women, given that HPV16 and HPV18 together account for $60-70 \%$ of such lesions. This protection would translate to a substantial reduction in medical and psychological morbidity and treatment together with a reduction in the related costs. The anticipated eventual reduction in the incidence of cervical cancers and its consequences would be anticipated to be at least as great. If the vaccine were widely administered to populations that historically are less likely to be screened regularly, the vaccine could prevent most of those serious infections that currently are not detected because of a lack of screening (49). The impact on subclinical and low-grade dysplasias would be expected to be more modest, as only a minority of these infections are attributable to HPV16 and HPV18 (or to HPV6/11/16/18, when considering the quadrivalent Merck vaccine infection) (50). Although these anticipated reductions in CIN2+ and invasive cervical cancer would be impressive, it must be noted that there would still be many serious
HPV infections against which the vaccine would not protect. For this reason, it will be essential to educate health care providers and patients about this limitation of the vaccine and to emphasize that it will be necessary for vaccinated women to follow current cervical cancer screening guidelines.

What about vaccination in developing countries, where $80 \%$ of cervical cancers occur, but where medical resources are relatively scarce? The vaccine probably has the potential to prevent several hundred thousand cancers annually, many of which affect relatively young women and therefore have an enormous impact on their life expectancies (11). However, vaccination in low-resource settings would probably be cost-effective only if the expense of vaccination were modest, especially in view of the long interval between infection and the development of invasive cancer. The history of hepatitis B vaccination suggests that it may take many years to achieve a cost-effective vaccination program in developing areas (51). Thus reducing the cost of vaccination would be a priority for the developing world. In the interim, a substantial reduction in the incidence of cervical cancer might be achievable in these settings, in less time than vaccination would lead to a reduction, by the alternate modality of a low-cost once- or twice-in-a-lifetime screen-and-treat approach to cervical cancer prevention $(52,53)$.

\section{Acknowledgments}

This work was supported by the Intramural Research Program of the NIH, National Cancer Institute, Center for Cancer Research.

Address correspondence to: Douglas R. Lowy, Laboratory of Cellular Oncology, Center for Cancer Research, National Cancer Institute, National Institutes of Health, Bethesda, Maryland 20892, USA. Phone: (301) 496-9513; Fax: (301) 480-5322; E-mail: drl@helix.nih.gov.
1. Pisani, P., Parkin, D.M., Munoz, N., and Ferlay, J. 1997. Cancer and infection: estimates of the attributable fraction in 1990. Cancer Epidemiol. Biomarkers Prev. 6:387-400.

2. Lowy, D.R., and Howley, P.H. 2001. Papillomaviruses. In Fields virology. D.M. Knipe and P.H. Howley, editors. Lippincott, Williams, \& Wilkins. Philadelphia, Pennsylvania, USA. 2231-2264.

3. Zur Hausen, H. 2002. Papillomaviruses and cancer: from basic studies to clinical application. Nat. Rev. Cancer. 2:342-350.

4. Bosch, F.X., Schiffman, M., and Solomon, D., editors. 2003. Future directions in epidemiologic and preventive research on human papillomaviruses and cancer. J. Natl. Cancer Inst. Monogr. 31:131.

5. Frisch, M. 2002. On the etiology of anal squamous carcinoma. Dan. Med. Bull. 49:194-209.

6. Gillison, M.L., and Shah, K.V. 2003. Chapter 9: role of mucosal human papillomavirus in nongenital cancers. J. Natl. Cancer Inst. Monogr. 31:57-65.

7. Schiffman, M., and Kjaer, S.K. 2003. Chapter 2 : natural history of anogenital human papillomavirus infection and neoplasia. J. Natl. Cancer Inst. Monogr. 31:14-19.

8. Gillison, M.L., and Lowy, D.R. 2004. A causal role for human papillomavirus in head and neck cancer. Lancet. 363:1488-1489.

9. Szentirmay, Z., et al. 2005. Human papillomavirus in head and neck cancer: molecular biology and clinicopathological correlations. Cancer Metastasis Rev. 24:19-34.

10. Pfister, H. 2003. Chapter 8: human papillomavirus and skin cancer. J. Natl. Cancer Inst. Monogr. 31:52-56.

11. Yang, B.H., Bray, F.I., Parkin, D.M., Sellors, J.W., and Zhang, Z.F. 2004. Cervical cancer as a priority for prevention in different world regions: an evaluation using years of life lost. Int. J. Cancer. 109:418-424.

12. Snijders, P.J., Steenbergen, R.D., Heideman, D.A., and Meijer, C.J. 2006. HPV-mediated cervical carcinogenesis: concepts and clinical implications. J. Pathol. 208:152-164

13. Wright, T.C., Jr., Cox, J.T., Massad, L.S., Twiggs, L.B., and Wilkinson, E.J. 2002. 2001 consensus guidelines for the management of women with cervical cytological abnormalities. JAMA. 287:2120-2129.

14. Bosch, F.X., Lorincz, A., Munoz, N., Meijer, C.J., and Shah, K.V. 2002. The causal relation between human papillomavirus and cervical cancer. J. Clin. Pathol. 55:244-265.

15. Munoz, N., et al. 2004. Against which human papillomavirus types shall we vaccinate and screen? The international perspective. Int. J. Cancer. 111:278-285.

16. Cates, W., Jr. 1999. Estimates of the incidence and prevalence of sexually transmitted diseases in the United States. American Social Health Association Panel. Sex. Transm. Dis. 26(4 Suppl.):S2-S7.

17. Baseman, J.G., and Koutsky, L.A. 2005. The epidemiology of human papillomavirus infections. J. Clin. Virol. 32(Suppl. 1):S16-S24.

18. Khan, M.J., et al. 2005. The elevated 10 -year risk of cervical precancer and cancer in women with human papillomavirus (HPV) type 16 or 18 and the possible utility of type-specific HPV testing in clinical practice. J. Natl. Cancer Inst. 97:1072-1079.

19. Littler, E., and Oberg, B. 2005. Achievements and challenges in antiviral drug discovery. Antivir. Chem. Chemother. 16:155-168.

20. Roden, R.B., Ling, M., and Wu, T.C. 2004. Vaccination to prevent and treat cervical cancer. Hum.
Pathol. 35:971-982.

21. Ehreth, J. 2005. The economics of vaccination from a global perspective: present and future. 2-3 December, 2004, Vaccines: all things considered, San Francisco, CA, USA. Expert Rev. Vaccines. 4:19-21.

22. Robbins, J.B., Schneerson, R., and Szu, S.C. 1995. Hypothesis: serum IgG antibody is sufficient to confer protection against infectious diseases by inactivating the inoculum. J. Infect. Dis. 171:1387-1398.

23. Kirnbauer, R., Booy, F., Cheng, N., Lowy, D.R., and Schiller, J.T. 1992. Papillomavirus L1 major capsid protein self-assembles into virus-like particles that are highly immunogenic. Proc. Natl. Acad. Sci.U.S. A. 89:12180-12184

24. Hagensee, M.E., Yaegashi, N., and Galloway, D.A. 1993. Self-assembly of human papillomavirus type 1 capsids by expression of the L1 protein alone or by coexpression of the L1 and L2 capsid proteins. J. Virol. 67:315-322.

25. Rose, R.C., Bonnez, W., Reichman, R.C., and Garcea, R.L. 1993. Expression of human papillomavirus type $11 \mathrm{~L} 1$ protein in insect cells: in vivo and in vitro assembly of viruslike particles. J. Virol. 67:1936-1944.

26. Kirnbauer, R., et al. 1993. Efficient self-assembly of human papillomavirus type $16 \mathrm{~L} 1$ and L1-L2 into virus-like particles. J. Virol. 67:6929-6936.

27. Rose, R.C., Reichman, R.C., and Bonnez, W. 1994. Human papillomavirus (HPV) type 11 recombinant virus-like particles induce the formation of neutralizing antibodies and detect HPV-specific antibodies in human sera. J. Gen. Virol. 75:2075-2079.

28. Roden, R.B.S., et al. 1996. In vitro generation and type-specific neutralization of a human papillomavirus type 16 virion pseudotype. J. Virol. 70:5875-5883. 
29. Breitburd, F., et al. 1995. Immunization with viruslike particles from cottontail rabbit papillomavirus (CRPV) can protect against experimental CRPV infection. J. Virol. 69:3959-3963.

30. Suzich, J.A., et al. 1995. Systemic immunization with papillomavirus L1 protein completely prevents the development of viral mucosal papillomas. Proc. Natl. Acad. Sci. U. S. A. 92:11553-11557.

31. Kirnbauer, R., et al. 1996. Virus-like particles of Bovine Papillomavirus type 4 in prophylactic and therapeutic immunization. Virology. 219:37-44.

32. Koutsky, L.A., et al. 2002. A controlled trial of a human papillomavirus type 16 vaccine. N. Engl. J. Med. 347:1645-1651.

33. Villa, L.L., et al. 2005. Prophylactic quadrivalent human papillomavirus (types 6, 11, 16, and 18) L1 virus-like particle vaccine in young women: a randomised double-blind placebo-controlled multicentre phase II efficacy trial. Lancet Oncol. 6:271-278.

34. Mao, C., et al. 2006. Efficacy of human papillomavirus-16 vaccine to prevent cervical intraepithelial neoplasia: a randomized controlled trial. Obstet. Gynecol. 107:18-27.

35. Harper, D.M., et al. 2004. Efficacy of a bivalent L1 virus-like particle vaccine in prevention of infection with human papillomavirus types 16 and 18 in young women: a randomised controlled trial. Lancet. 364:1757-1765.

36. De Villiers, E.M., Fauquet, C., Broker, T.R., Bernard, H.U., and zur Hausen, H. 2004. Classification of papillomaviruses. Virology. 324:17-27.

37. Roden, R.B.S., et al. 1996. Assessment of the serological relatedness of genital human papillomaviruses by hemagglutination inhibition. J. Virol. 70:3298-3301.
38. Greer, C.E., et al. 1995. Human papillomavirus (HPV) type distribution and serological response to HPV 6 virus-like particle in patients with genital warts. J. Clin. Microbiol. 33:2058-2063.

39. [No authors listed]. 2001. FDA vaccines and related biological products advisory committee. Center for Biologics Evaluation and Research meeting \#88. November 28-29. Bethesda, Maryland, USA. http://www.fda.gov/ohrms/dockets/ac/cber01. htm\#Vaccines.

40. Stoler, M.H. 2003. Human papillomavirus biology and cervical neoplasia: implications for diagnostic criteria and testing. Arch. Pathol. Lab. Med. 127:935-939.

41. Harro, C.D., et al. 2001. Safety and immunogenicity trial in adult volunteers of a human papillomavirus 16 L1 virus-like particle vaccine. J. Natl. Cancer Inst. 93:284-292.

42. Barr, E. 2005. Gardasil HPV vaccine. Presented at the Centers for Disease Control and Prevention, National Immunization Program meeting of the Advisory Committee on Immunization Practices. February 10-11. Atlanta, Georgia, USA.

43. Barr, E. 2006. Garadasil HPV vaccine. Presented at the Centers for Disease Control and Prevention, National Immunization Program meeting of the Advisory Committee on Immunization Practices. February 21-22. Atlanta, Georgia, USA.

44. Dubin, G. 2005. Enhanced immunogenicity of a candidate human papillomavirus (HPV) 16/18 L1 virus like particle (VLP) vaccine with novel AS04 adjuvant in preteens/adolescents. Presented at the 45th Annual International Conference on Antimicrobial Agents and Chemotherapy (ICAAC). December 16-19. Washington, DC, USA.
45. Dubin, G. 2006. Cervarix HPV vaccine. Presented at the Centers for Disease Control and Prevention, National Immunization Program meeting of the Advisory Committee on Immunization Practices. February 21-22. Atlanta, Georgia, USA.

46. Nardelli-Haefliger, D., et al. 2003. Specific antibody levels at the cervix during the menstrual cycle of women vaccinated with human papillomavirus 16 virus-like particles. J. Natl. Cancer Inst. 95:1128-1137.

47. Stanberry, L.R., et al. 2002. Glycoprotein-D-adjuvant vaccine to prevent genital herpes. $N$. Engl. J. Med. 347:1652-1661.

48. Garnett, G.P. 2005. Role of herd immunity in determining the effect of vaccines against sexually transmitted disease. J. Infect. Dis. 191(Suppl. 1):S97-S106.

49. Freeman, H., and Wingrove, B. 2005. Excess cervical cancer mortality: a marker for low access to health care in poor communities. National Cancer Institute, Center to Reduce Cancer Health Disparities. Rockville, Maryland, USA. http://crchd.nci.nih. gov/initiatives/\#Reducing. 79 pp.

50. Clifford, G.M., et al. 2005. Human papillomavirus genotype distribution in low-grade cervical lesions: comparison by geographic region and with cervical cancer. Cancer Epidemiol. Biomarkers Prev. 14:1157-1164.

51. Ulmer, J.B., and Liu, M.A. 2002. Ethical issues for vaccines and immunization. Nat. Rev. Immunol. 2:291-296.

52. Goldie, S.J., et al. 2005. Cost-effectiveness of cervical-cancer screening in five developing countries. N. Engl.J. Med. 353:2158-2168.

53. Schiffman, M., and Castle, P.E. 2005. The promise of global cervical-cancer prevention. N. Engl. J. Med. 353:2101-2104 